\title{
LAS IDENTIDADES TRANSITORIAS Estrategias de socialización de los residentes europeos en la Comunidad Valenciana
}

\author{
TRANSITIONAL IDENTITIES \\ Socialization strategies of European residents in the Valencian Community
}

Francisco José FrancÉs García francisco.frances@ua.es
Universidad de Alicante. España

Óscar Antonio Santacreu Fernández oscar.santacreu@ua.es

Universidad de Alicante. España

\begin{abstract}
RESUMEN
El artículo plantea un análisis en torno a las claves explicativas del proceso de formación de capital social y las estrategias de inserción en la sociedad civil española de los extranjeros europeos residentes en la Comunidad Valenciana. La hipótesis de trabajo plantea la existencia de dos discursos diferenciados (integración y coexistencia). Los resultados muestran cómo las variables "edad" y "posición laboral" determinan en gran medida la adscripción a uno u otro discurso, manteniendo sin embargo elementos comunes, como son la concepción individual del proceso de inserción en la sociedad civil y la consideración instrumental de la nacionalidad. Esto revela que el proceso de construcción de capital social de los extranjeros europeos compone un paradigma explicativo diferente al de los procesos de inmigración extracomunitaria. El artículo finaliza con un análisis dimensional de diferentes elementos propios del capital social vinculado a la realidad de la población estudiada.
\end{abstract}

\section{Palabras Clave}

Capital social; Integración social; Población inmigrante europea; Sociedad civil.

\section{Abstract}

The article presents an analysis around the key explanations that account for the process of social capital formation and strategies for integration into Spanish society of European foreign residents in the Region of Valencia. The working hypothesis handles the existence of two distinct discourses, classified as integration discourse and coexistence discourse. The results show how the variables "age" and "job position" largely determine the assignment to one or another discourses, while maintaining common elements such as individual conception of the process of integration into civil society and the instrumental account of nationality. This reveals that the process of building social capital of European foreigners make up a very different paradigm explaining the nature of the processes underlying immigration from outside. The article ends with a dimensional analysis of different elements of social capital and its relationship to the reality of European foreign residents in the Valencian Community.

\section{KEYWORDS}

Civil Society; European Immigrant Population; Social Capital; Social Integration. 


\section{INTRODUCCIÓN}

Hablar sobre la relación existente entre capital social y flujos migratorios resulta ciertamente arriesgado. Arriesgado, en principio, porque cabría pensar que difícilmente un texto de esta brevedad puede aportar elementos adicionales a los ya tratados desde la amplia bibliografía existente en este campo de investigación. Arriesgado también porque, dada la enorme variabilidad del fenómeno migratorio tanto en su naturaleza como en los territorios y las poblaciones que lo protagonizan, establecer lecturas generalistas nos puede abocar probablemente a una excesiva simplificación de la realidad social.

Pero estas premisas, que en principio podrían considerarse factores limitantes para la reflexión en torno a cuestiones de esta naturaleza, muestran sin embargo paradojas que abren importantes retos para la investigación social. El primer hecho en el que descansa la paradoja radica en el extraordinario dinamismo de los procesos migratorios, que provoca que cualquier análisis de la realidad social tenga una rápida fecha de caducidad. Los flujos cambiantes, así como el creciente peso cuantitativo de los contingentes de población que varían su residencia, suponen un objeto de interés constante tanto desde el ámbito institucional como desde la academia. En segundo lugar, cada vez parece más claro que el fenómeno migratorio ha trascendido en mucho a la mera dimensión económica o demográfica que estos procesos sociales incorporan en los territorios de acogida. En efecto, aunque estas facetas continúan centrando el interés investigador, no cabe sino hablar de una pluralidad de aspectos de la realidad social sobre los que el hecho migratorio impacta, en muchos casos transversalmente. Siendo central la necesidad de conocimiento sobre las consecuencias socioeconómicas de los procesos migratorios, es indudable su influencia en otros aspectos de la dinámica social como la cultura, los comportamientos políticos y democráticos, la educación, las formas de convivencia, o las maneras en que se vertebra una sociedad civil caracterizada, cada vez más, por el considerable aumento de poblaciones con usos y costumbres muy heterogéneas.

Precisamente esta última esfera, la de la sociedad civil, elemento inherente a la vida social en cualquier comunidad, es la que nos va a ocupar en las siguientes páginas. La sociedad civil y la cuestión de la ciudadanía como constructos teóricos poseen un indudable interés investigador. Ahora bien, aunque hacemos un uso habitual de estos vocablos en el lenguaje investigador, en ocasiones no descansa la suficiente reflexión sobre las realidades y prácticas concretas en que cristalizan estos conceptos. Difícilmente podemos escapar a la idea de que los procesos migratorios han transformado, a la vez que aumentado, la diversidad de las formas en que se articulan la ciudadanía, las relaciones entre los diferentes segmentos de población, o los intereses y prioridades que dichos segmentos incorporan a la formación del capital social comunitario. La incipiente pero progresiva incorporación de los inmigrantes extranjeros a las esferas de la participación social y política ha acrecentado los cambios que los flujos migratorios 
están produciendo en la semántica tradicional de la ciudadanía (Miravet 2006). El hecho migratorio supone por lo tanto un reto crucial en la transformación de la sociedad civil, a la vez que esta incide también de manera apreciable y contribuye a explicar las direcciones de los movimientos migratorios, las razones y el número de personas involucradas en ellos. En España existe ya una amplia literatura en torno al valor del asociacionismo inmigrante como factor conformador del proceso de integración social para la población extranjera (Aparicio y Tornos 2010; Martín 2004; Migallón 2004; Morales et al. 2008; Morell 2005; Torres 2005; Veredas 2003; Vidal et al. 2007). Pero más allá de los indicadores de sociabilidad formal basados en la membresía asociativa, parece recomendable adentrarse en otras dimensiones que sin duda también dan cuenta del proceso de integración en la sociedad civil de acogida. Itinerarios cambiantes de inserción, mantenimiento o modificación de las tradiciones culturales, transformación de las redes sociales y afectivas, implicación en espacios de participación social, o uso de los medios de comunicación constituyen elementos que proporcionan claves muy valiosas para la configuración del capital social de la población inmigrante extranjera, y estas claves están presentes en sus discursos de manera más o menos explícita.

La fundamentación teórica del concepto de capital social es bien profusa. Como bien señala Fine (2001), al tratar de hacer una revisión bibliográfica sobre el capital social, de inmediato uno se ve a sí mismo persiguiendo a un objetivo móvil cuya principal característica es reproducirse a una velocidad que desafía cualquier capacidad humana para seguirlo. Esto no pretende ser una excusa, sino más bien una llamada de alerta sobre la magnitud del objeto de estudio en cuestión. No es el objeto del presente artículo la conceptualización del capital social, pero conviene acercarse a las aportaciones teóricas y empíricas que proponen elementos analíicos de utilidad en la comprensión de las relaciones entre capital social y procesos migratorios.

En la literatura moderna, autores como Coleman o Putnam constituyen sin duda soportes recurrentes para abordar la noción de capital social. En el caso de Coleman (1988) la pertenencia a grupos, y la implicación en las redes sociales desarrolladas dentro de estos, así como las relaciones sociales que surgen de la pertenencia, puede ser utilizada para mejorar la posición de los actores en una variedad enorme de campos, y facilitan ciertas acciones de los actores (sean individuales o colectivos) dentro de la estructura social en forma de capital social. El desarrollo de las redes sociales dependería al mismo tiempo del sentimiento subjetivo individual (reconocimiento, respeto y comunalidad) y de la seguridad permitida por el resto de sujetos de la comunidad. Para Putnam (1993; 1995; 2000), en realidad, el concepto de capital social expresa la esencia sociológica de la vitalidad comunitaria, y en su definición aporta tres componentes básicos: normas y obligaciones morales, valores sociales (especialmente confianza) y redes sociales (especialmente asociaciones voluntarias). En relación a estas ideas, dentro del campo específico de las migraciones, algunas corrientes investigadoras conciben el capital social como uno de los factores explicativos de las migraciones. 
El capital social contribuiría a comprender no solo los motivos de la migración sino también la dirección de los flujos migratorios, su carácter selectivo y la consolidación de los contingentes extranjeros en los nuevos territorios. Desde esta perspectiva, Massey y Espinosa (1997) hallaron que el capital social, mediante el soporte institucional, las asociaciones y la colaboración mutua entre los miembros de la comunidad, ayuda a explicar hacia dónde se dirigen los movimientos migratorios, sus razones y el número de personas implicadas en los procesos. En consonancia con esta línea y para el caso español, podemos destacar la investigación coordinada por Martín Hernández (2007), que a partir de su estudio sobre capital social e inmigración en la provincia de Teruel, concluye que en dicho ámbito geográfico, con unas elevadas tasas de despoblación y de abandono, la inmigración puede ser una alternativa vital no solo en términos de capital humano, sino también como agentes del capital social, rescatando el concepto como un claro indicador de riqueza y desarrollo comunitario.

En paralelo a las corrientes teóricas apuntadas, Bourdieu $(1985 ; 1998)$ destaca los beneficios instrumentales de los individuos en virtud de su participación en grupos, y en la deliberada construcción de la sociabilidad con el propósito de crear este recurso a través de las redes sociales en el nuevo territorio de acogida. Ligado a esta idea, algunas de las aplicaciones más significativas de la teoría del capital social en el campo de las migraciones han sido las realizadas por Portes (1995; 1998). Sus investigaciones muestran la importancia de las redes sociales como fuente de recursos materiales e inmateriales (acceso a la vivienda y al empleo, apoyo afectivo, etc.) en los procesos de inserción social de la población inmigrante dentro de la sociedad de acogida, procesos que oscilan entre el éxito y el fracaso. En sus estudios sobre las diferentes comunidades de inmigrantes en los Estados Unidos, Portes concluye que el capital social es diferente en función de la nacionalidad o grupo étnico al que pertenecen y, a la vez, dependiente de la estructura social del territorio al que llegan.

En definitiva, lo cierto en cualquier caso es que, dada la heterogeneidad del propio concepto, diferentes formas de entender el capital social nos llevan necesariamente a diferentes formas de medirlo. Ajustando el planteamiento investigador de este texto, entenderemos el capital social como un concepto de dimensiones múltiples que expresa la relación multidireccional entre confianza, participación cívica, asociatividad, compromiso social y reciprocidad, potenciando las capacidades individuales y colectivas de acción. Una definición que, por otra parte viene a ser una consideración afín a la que plantea el Banco Mundial (1999).

Tras lo visto, al hablar de inserción o integración parece claro que nos situamos ante un campo de análisis ligado a la idea de capital social. Pero en el caso del análisis que nos ocupa el interés se centra en la identificación de claves explicativas más allá de la sociabilidad formal o la asociatividad, cuestiones manejadas como centrales 0 motrices en los teóricos del capital social, y protagonistas de gran parte del corpus de producción en este ámbito investigador. Las estrategias de inserción de la población 
extranjera hacen referencia por supuesto a estos elementos propios del capital social individual o colectivo pero más allá, la configuración de los discursos se alimenta también de otras claves explicativas, de carácter difuso en ocasiones, pero definitorias en la obligada reconfiguración del proceso identitario vinculado a la construcción del sentido de ciudadanía en la nueva sociedad de acogida.

Vamos a tratar todas estas cuestiones desde una realidad concreta, tanto poblacional como territorial, que creemos de interés por cuanto constituye un fenómeno, en nuestra opinión, tratado de manera insuficiente desde la labor investigadora; el objetivo va a ser el análisis de las distintas estrategias de inserción en la sociedad civil de los extranjeros pertenecientes a la Unión Europea (UE15) residentes en la Comunidad Valenciana. Se trata de un marco territorial donde, como podremos comprobar, el impacto de la población extranjera europea ha reconfigurado sensiblemente el concepto de la ciudadanía. Supone por lo tanto un "laboratorio de las migraciones" (Gómez 2006) en el que hallamos una serie de procesos sociales específicos que, aunque tratados únicamente desde el punto de vista de la participación formal, van siendo objeto de una incipiente atención investigadora (Gómez 2006; Jabbaz et al. 2004; Massanet y Santacreu 2010; Simó et al. 2005).

El análisis de la aportación de los inmigrantes al capital social de la Comunidad Valenciana, en términos de participación social, exige en primer lugar justificar la elección de este territorio para explorar las estrategias de inserción en la sociedad civil del contingente de población elegido.

\section{EL FENÓMENO MIGRATORIO DE LOS EXTRANJEROS EUROPEOS RESIDENTES EN LA Comunidad Valenciana}

Que la Comunidad Valenciana sea el territorio donde analizar las formas mediante las que los extranjeros residentes europeos inciden en la transformación de la sociedad civil de acogida no es casual. La Comunidad Valenciana es un espacio de encuentro multicultural que, por sus condiciones económicas, geográficas y de ocio, se ha convertido en un espacio atractivo para el turismo, la jubilación, el trabajo y el estudio. Ha llegado a ser, en poco tiempo, un espacio pionero en cuanto a los procesos de movilidad humana con las implicaciones de todo tipo que esto conlleva.

Como podemos observar en la figura 1, la evolución de la presencia de residentes extranjeros europeos en la Comunidad Valenciana es significativa. En los últimos doce años se ha multiplicado por cuatro este colectivo de población, que en la actualidad constituye un conjunto de unas 300.000 personas (un $5,65 \%$ del total de la población de la Comunidad Valenciana).

Dentro del territorio valenciano, la presencia de esta población no es, ni mucho menos, homogénea. Ciertamente, la provincia de Alicante emerge como el centro de 
Figura 1.

Evolución de la población extranjera UE

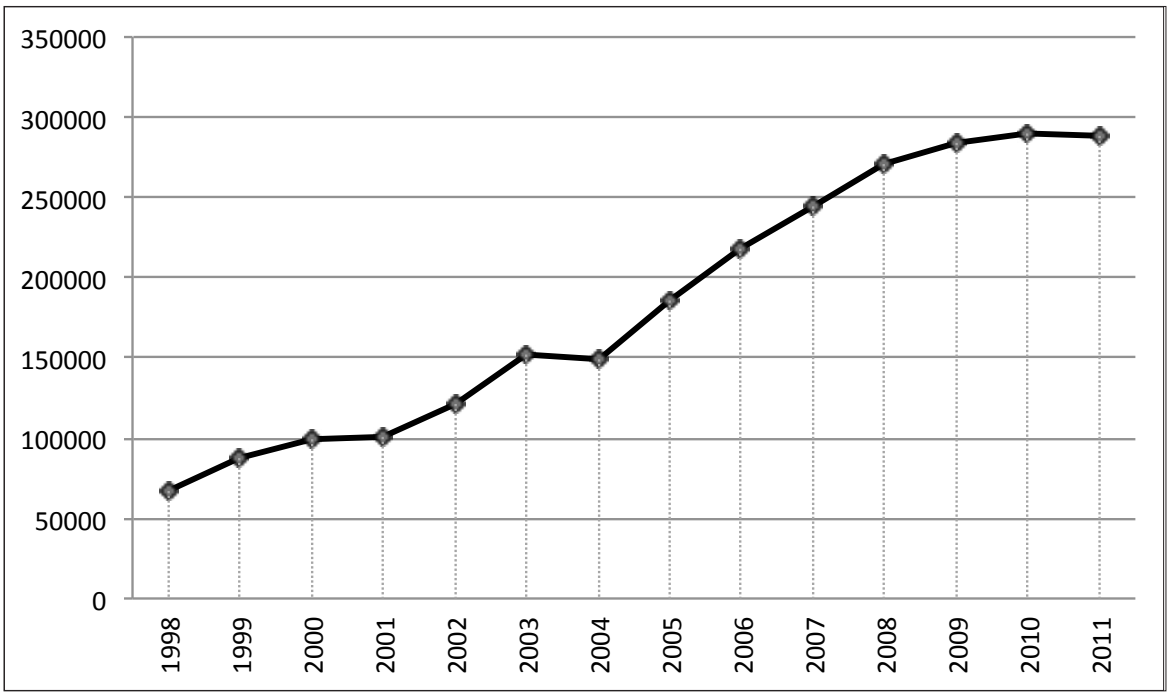

Fuente: INE, Padrón Municipal, series de población.

atracción de los residentes europeos comunitarios, concentrando el $81,86 \%$ del total presente en la Comunidad Valenciana (236.492 extranjeros europeos en la provincia de Alicante, 41.969 en la de Valencia y 10.429 en la de Castellón). Esta cifra, para el caso de Alicante, supone el $12,23 \%$ de toda la población de la provincia.

Así, la Comunidad Valenciana, y la provincia de Alicante en particular, se hallan a la cabeza estatal en lo relativo a la presencia de extranjeros europeos residentes.

A la luz de los datos que presentan las estadísticas, no cabe calificar el proceso migratorio que nos ocupa como de Estado a Estado, sino como un flujo transnacional en el que la movilidad se realiza hacia zonas concretas del Estado de acogida. Así, podemos hablar de zonas que presentan características propias (por su especial crecimiento económico, por sus condiciones de vida, por la existencia asentada ya de un contingente de población extranjera, etc.) que hacen atractivo el fenómeno del cambio de residencia. Para el caso de España podemos apreciar cómo los contingentes de residentes extranjeros europeos se concentran en el arco mediterráneo (Figura 2). Las comunidades autónomas con mayor presencia dentro del Estado son la Comunidad Valenciana, seguida de Andalucía y Cataluña. Pero si cabe hablar de zonas de destino comunes, también ocurre lo mismo al analizar los países de origen. Para el territorio valenciano, los datos del padrón municipal de 2011 muestran la importancia del colectivo de ingleses (144.414 personas), alemanes (42.511) y, en menor medida, franceses, italianos y holandeses, que habitan, en relación a las zonas de influencia de la Europa comunitaria. 
Figura 2.

Población extranjera UE(15) por CC.AA.

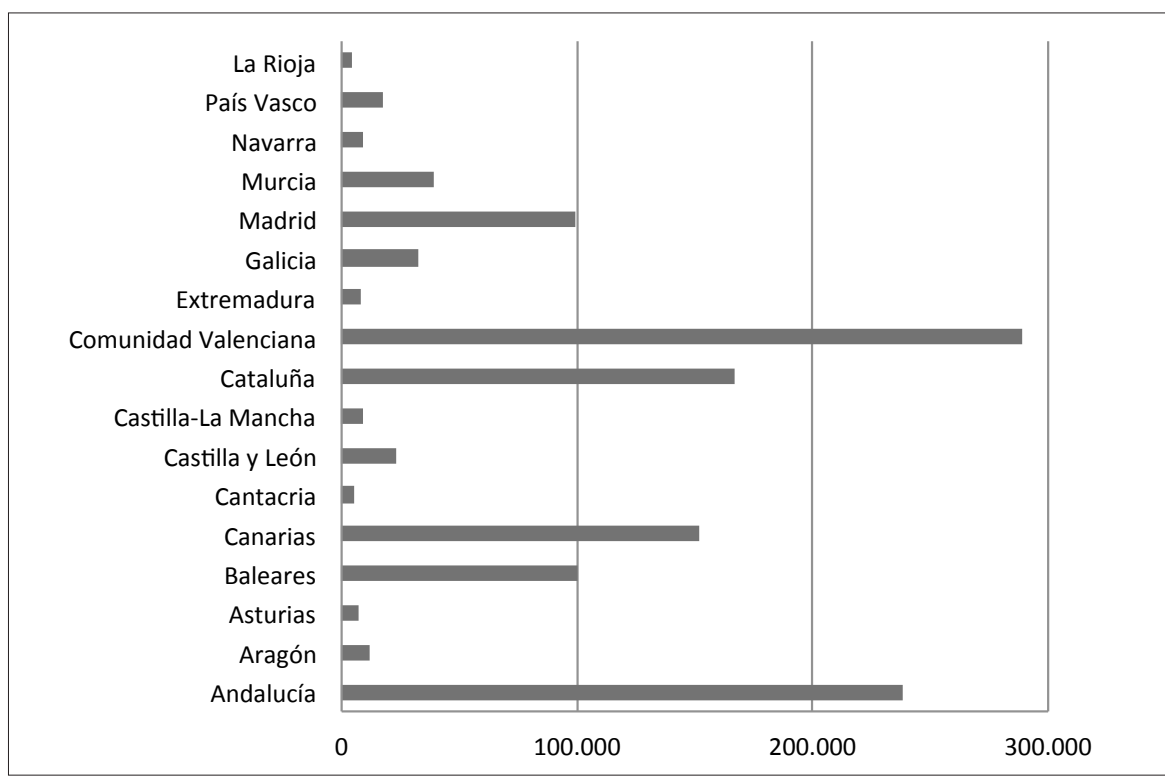

Fuente: Padrón municipal 2011. Instituto Nacional de Estadística.

Si bien es cierto que las situaciones del cambio residencial en el caso de los extranjeros europeos se polarizan en torno a una serie de denominadores comunes, podemos apreciar cómo es también posible identificar diferencias sustanciales en la naturaleza de las decisiones que dan lugar a este fenómeno, ya al margen de la nacionalidad de origen. Estos elementos configuran fracturas en los procesos de integración de esta población dentro de la sociedad civil del territorio de acogida. Y como podremos ver más delante, en torno a las estrategias de inserción surge una variable que da cuenta en buena medida de las diferencias existentes, influyendo de manera determinante en las actitudes y predisposiciones de los residentes extranjeros europeos en relación a la formación de capital social. Esa variable es la edad.

La distribución de la edad en los residentes extranjeros europeos (Figura 3) viene a decirnos que cabe hablar de una concentración de la población en los grupos de edad que podríamos situar en la salida del período laboral de estas personas. Esta cuestión nos lleva a identificar una falla creciente entre la situación de la población extranjera europea que se halla activa en el mercado laboral y aquella que ya se sitúa al margen de éste. Precisamente esta falla permitirá explicar gran parte de las diferencias presentes en este colectivo en relación al tema que nos ocupa. 
Figura 3.

Población extranjera UE(15) en la Comunidad Valenciana por grupos de edad.

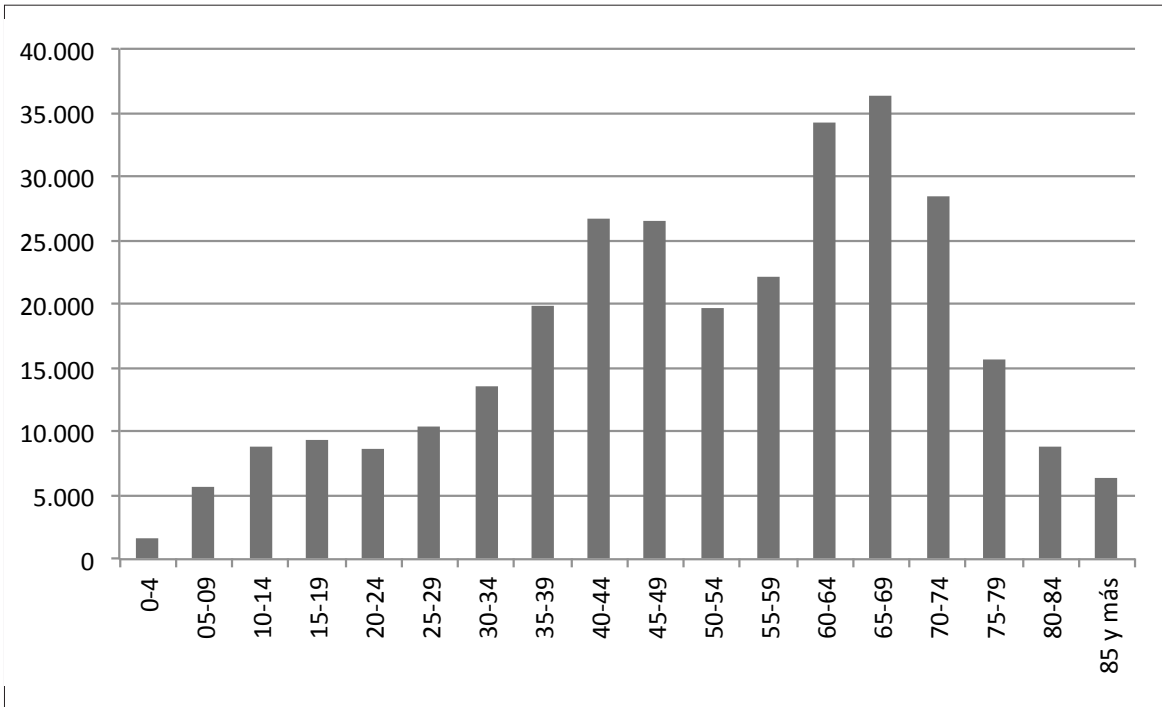

Fuente: Padrón municipal 2011. Instituto Nacional de Estadística.

Como se puede intuir, todos estos contrastes internos del contingente extranjero poseen una recepción plural, diversa, e incluso divergente en la sociedad receptora, a la vez que plantea actitudes diferentes en torno a la significación que esta población extranjera atribuye al sentido de ciudadanía y a su papel en los procesos de participación social en el nuevo territorio que habita, cuestiones que en definitiva soportan en gran medida la idea de capital social sobre la que pretendemos incidir.

Por ello a continuación nos centraremos en este colectivo para exponer, a través del análisis de las entrevistas realizadas con sujetos de diferentes nacionalidades y edades, sus estrategias de inserción, sus relaciones sociales y las características de su participación social.

\section{OBjetivos, Metodología e hiPótesis de INVESTIGACIÓN}

Los objetivos de nuestro estudio se insertan en dos ámbitos principalmente: el análisis de las relaciones entre capital social e integración social de los extranjeros europeos y, en segundo lugar, el análisis del papel que juegan las redes sociales y la transformación de las señas identitarias en el proceso de integración de los extranjeros dentro del nuevo 
territorio. Para ello emplearemos las dimensiones propuestas por Woolcock (1998), así como algunos de los indicadores de estas dimensiones empleados por Moyano (2006), adaptados a nuestro ámbito de investigación.

Desde diversas corrientes de la nueva sociología del desarrollo, el capital social ligado a los procesos migratorios se articula a partir de dos dimensiones fundamentales que combinan tanto el nivel macro como micro: embeddedness (enraizamiento o inserción) y autonomy (autonomía). Basándose en estos dos conceptos, y en la combinación de ambas dimensiones, Woolcock propone un modelo-síntesis compuesto por cuatro dimensiones que en nuestro caso sirven para el análisis de las dinámicas migratorias. Los indicadores utilizados en nuestro análisis sobre las estrategias de inserción de la población extranjera europea en la sociedad civil del territorio de acogida se muestran en el Cuadro 1.

Estos indicadores constituyen la matriz del análisis para la investigación. Contribuyen a organizar la información del trabajo de campo y a valorar los resultados, aunque debemos anticipar que las hipótesis investigadoras son transversales en el sistema de indicadores propuesto, lo que llevará a establecer lecturas sintéticas que combinen una o más dimensiones para la comprobación de cada una de las hipótesis. Por lo tanto las conclusiones se guiarán por el conjunto de hipótesis, más allá de los indicadores utilizados.

Para su estudio, además de la utilización de fuentes secundarias con datos del Padrón municipal de habitantes de 2011 y el Censo, sobre todo se ha recurrido a fuentes primarias. El uso de datos primarios en nuestro análisis emplea una metodología de corte cualitativo, basada en el análisis de discurso sobre entrevistas semiestructuradas realizadas a personas extranjeras europeas (UE15) en el contexto de dos investigaciones: "La participación social de los inmigrantes en la Comunidad Valenciana y su contribución a la formación de capital social"1, realizada en 2007, y la investigación "Competencia intercultural e integración de los residentes extranjeros europeos en la Comunidad Valenciana. Un análisis diagnóstico y prospectivo de los estereotipos económicos, sociales y políticos, y su efecto sobre la inmigración como pull factor"2, realizada en 2005. En el contexto de estas investigaciones se llevaron a cabo entrevistas con un diseño de muestreo intencional por cuota. La afijación de las cuotas estuvo definida por la variabilidad en términos de nacionalidad (alemanes, británicos, italianos y franceses), tiempo de residencia en España (más de 5 años o no), consideración de su situación activa o no en el mercado laboral, género y grupos de edad. El tamaño muestral se guió

\footnotetext{
${ }^{1}$ Proyecto financiado por la Conselleria d'Empresa, Universitat i Ciència de la Generalitat Valenciana, con referencia GV05/171.

${ }^{2}$ Proyecto financiado por la Conselleria de Cultura, Educación y Deporte de la Generalitat Valenciana, con referencia (GV04B/621).
} 


\section{Cuadro 1.}

Dimensiones e indicadores de la integración y el capital social en la población extranjera europea.

\begin{tabular}{|c|c|}
\hline Integración intracomunitaria & $\begin{array}{l}\text { - Participación de los inmigrantes en } \\
\text { asociaciones orientadas a la población } \\
\text { extranjera. } \\
\text { - Mantenimiento de señas de identidad y/o } \\
\text { tradiciones. } \\
\text { - Redes sociales en los países de origen. } \\
\text { - Consumo de los medios de comunicación de } \\
\text { su propia nacionalidad. }\end{array}$ \\
\hline Conexión extracomunitaria & $\begin{array}{l}\text { - Participación de los extranjeros en } \\
\text { organizaciones distintas a las de su grupo de } \\
\text { pertenencia (participación en asociaciones } \\
\text { autóctonas). } \\
\text { - Adopción de nuevas señas de identidad y/o } \\
\text { tradiciones. } \\
\text { - Participación sociopolítica en el nuevo } \\
\text { territorio. } \\
\text { - Consumo de los medios de comunicación de } \\
\text { la nueva comunidad. }\end{array}$ \\
\hline Sinergia institucional & $\begin{array}{l}\text { - Interacción y cooperación entre las distintas } \\
\text { redes y colectivos de extranjeros. } \\
\text { - Colaboración entre colectivos de extranjeros } \\
\text { e instituciones públicas. }\end{array}$ \\
\hline Eficiencia organizativa & $\begin{array}{l}\text { - Reconocimiento social e institucional de los } \\
\text { extranjeros por parte de la Administración } \\
\text { Pública. } \\
\text { - Percepción de la eficiencia y calidad de los } \\
\text { servicios prestados por las instituciones. } \\
\text { - Valoración individual o colectiva de la } \\
\text { trayectoria y posibilidades de inserción } \\
\text { socio-laboral. }\end{array}$ \\
\hline
\end{tabular}

Fuente: Elaboración propia, a partir de Woolcock (1998).

por la redundancia y saturación de la información, de lo que resultaron un total de 32 entrevistas.

El planteamiento investigador se halla guiado por el establecimiento de las hipótesis de trabajo que han organizado el análisis del material de campo. Acorde con lo planteado en la introducción, una primera hipótesis nos situaría en la idea de que las estrategias de inserción de la población extranjera europea en la Comunidad Valenciana siguen claves explicativas distintas a la de los extranjeros de otras procedencias. Este proceso 
diferenciado está presidido por la "laxitud de señas identitarias" habituales en los estudios migratorios, como son la nacionalidad, la lengua o las tradiciones (H1). La explicación a esta difuminación identitaria viene explicada en gran medida por el hecho de que "la estrategia de socialización de los extranjeros europeos posee un claro componente individual", al margen de las redes sociales de la población de su misma procedencia o ya existentes en el territorio de acogida ( $\mathrm{H} 2$ ). A pesar de estos elementos comunes en la población extranjera europea, una tercera hipótesis nos lleva a plantear la existencia de "diferencias internas significativas en el proceso de integración en función de la edad" de llegada de la persona al nuevo territorio $(\mathrm{H} 3)$.

\section{Discusión de RESULtAdos. ANÁlISIS dE LAS ESTRATEGIAS dE SOCIALIZACIÓN DE LOS RESIDENTES EUROPEOS COMUNITARIOS EN LA SOCIEDAD CIVIL}

En torno al impacto social de la presencia creciente de residentes extranjeros europeos en la Comunidad Valenciana, lo primero que podemos resaltar de los resultados del análisis es la identificación de dos grandes discursos claramente diferenciados. A través de estos dos posicionamientos los sujetos han explicitado dos orientaciones que les permiten autodefinir la razón de ser en la decisión de su residencia actual y los argumentos a través de los cuales desarrollan las percepciones vinculadas con la sociedad civil receptora. En este sentido se trata de dos posiciones no opuestas pero tampoco complementarias.

Un primer discurso es el que podríamos denominar el "discurso de la integración", enunciado y protagonizado básicamente por sujetos con un perfil de edad joven o adulta, pero en cualquier caso vinculados 0 en proceso de vinculación en la esfera productiva y laboral. Este discurso viene caracterizado básicamente por el reconocimiento expreso de la necesidad y el deber de los ciudadanos que acuden a residir a otro estado de integrarse de la mejor forma posible en la cultura y la forma de vida del país de acogida, tanto en lo referente a costumbres o hábitos como en las tradiciones culturales, o también el aprendizaje de la lengua de la comunidad a la que acude, uno de los elementos axiales que se atribuyen al ámbito de la integración cultural (Braun 2010). Nos hallamos por lo tanto ante una postura asimilacionista; que cataliza percepciones mucho más universalistas (relaciones con los españoles) en la interacción con la población de acogida que particularistas, esto es, relaciones principalmente con inmigrantes de su propio país (Díez Nicolás 2005:280)

"... no es que la sociedad se haga para mi, sino que si yo voy a un país, quiero ir a un país, intento al máximo adaptarme". (Hombre alemán, 45 años).

“...es España, entonces necesito hablar con ustedes en español, vivir como español no 
como un inglés. No me gusta un club de ingleses, no me gustan clubs, no me gustan las organizaciones". (Hombre inglés, 42 años).

Cuando vinimos a España, no queríamos vivir como en Inglaterra, quisimos vivir como españoles, hablar con los españoles de cosas españolas, y todo. (Mujer inglesa, 50 años).

Creo que hay que hacer un pequeño esfuerzo, integrarte un poco. No me gustan esos clanes cerrados. (Mujer francesa, 35 años).

Frente a este discurso podemos identificar otro enfoque que podríamos calificar como el "discurso de la coexistencia". Este discurso ya no mantiene como eje fundamental de argumentación el deber de integración de la población que acude a un nuevo territorio, sino que incide en el derecho de esa población a coexistir dentro del territorio con la población autóctona sin tener que adoptar necesariamente nuevos patrones culturales que les conduzcan a la integración. Cabe hablar en este sentido de una "aculturación selectiva" (Favell 2003), esto es, la obtención de lo que necesitan en el nuevo sistema social de acogida, pero sin tener que sacrificar sus propios hábitos y prácticas culturales. Encontramos aquí una caracterización y una categorización de sujetos distintas a las del discurso de la integración. En los posicionamientos propios de este discurso encontramos una población con un perfil de edad adulta o avanzada, que ya ha salido del mercado laboral y desarrolla una existencia más o menos ociosa en el nuevo lugar de residencia, favorecida generalmente por el hecho de que estos sujetos poseen una posición socioeconómica que podríamos calificar como buena o muy buena. En este discurso, la integración, la participación cívica y la integración social no constituyen una necesidad, sino en cualquier caso una decisión personal con motivaciones de carácter más cultural o vivencial que instrumental.

Vivimos en Alicante pero es como si viviéramos en Italia. (Hombre italiano, 59 años).

Conozco a muy pocas personas, y con muy pocas de ellas me veo regularmente. Pero amistad, no sé. Será porque empecé a conocer a estas personas cuando ya tenían una edad, o sea a los 42, ahora tengo 49, y cada vez es más complicado crear una amistad con la edad." (Hombre inglés, 49 años).

A pesar de las grandes diferencias entre ambos discursos, de las que nos ocuparemos en detalle más adelante, podemos sin embargo encontrar elementos comunes que ayudarán a identificar claves explicativas para los objetivos que nos hemos marcado. El primer nexo común de ambos posicionamientos es la valoración satisfactoria del cambio de residencia al acudir a la Comunidad Valenciana. La gran mayoría de los sujetos entrevistados evalúan positivamente la decisión de haber venido, independientemente del motivo del traslado o de la situación socioeconómica que alcanzan en España. Parece 
que un elemento determinante en esta valoración positiva del cambio de residencia es el hecho de que en ningún momento perciben rechazo por parte de la población española. Más bien al contrario, reconocen un esfuerzo por parte de los españoles para intentar desarrollar buenas relaciones entre ambos contingentes. Ciertamente, en opinión de los residentes extranjeros el estatus de ciudadanos comunitarios ayuda en gran medida a la aceptación de la población española, sobre la que proyecta un trato diferencial hacia los inmigrantes en función del lugar de procedencia de estos, pero no por ser de distinta nacionalidad.

Hay varios tipos de extranjeros. Yo no me identifico, ni tengo los mismos problemas que uno que viene de Marruecos... no es lo mismo ser de la Unión Europea que ser un emigrado. (Mujer francesa, 35 años).

Un indicio de que la posesión de otra nacionalidad comunitaria no es en ningún caso motivo de rechazo o discriminación lo muestra el hecho de que otro denominador común de los extranjeros objeto de la investigación es el mantenimiento de la nacionalidad de origen. En este sentido, creemos importante resaltar que la resistencia al cambio de nacionalidad y la decisión de mantener la nacionalidad de origen, aun estableciendo residencia definitiva en España, no parece responder a una cuestión de reforzamiento de la identidad o a un intento por mantener lazos de identificación con la comunidad de origen, lo cual difiere de la función efectiva de la nacionalidad como elemento identitario que parece recogerse en procesos migratorios de origen extracomunitario (Morales et al., 2008). La mayor parte de los residentes europeos otorgan a la nacionalidad un carácter instrumental, pragmático, a través de lo que podríamos denominar un cálculo de costo-beneficio en virtud del que determinan cuál es la nacionalidad que les aporta más ventajas en su vida cotidiana. Este posicionamiento explica en gran medida que generalmente opten por mantener la nacionalidad del país de origen, ya que esta les habilita para disponer de mayores prestaciones sociales y económicas que las que le otorgaría la asunción de la nacionalidad española.

Yo soy alemán, y mantengo la nacionalidad alemana, o sea, no la cambié por razones, inicialmente, más bien sociales, porque hay más prestaciones sociales, sanitarios, etc, ¿eh?, que aquí en España. (Hombre alemán, 55 años).

Dentro de esta visión instrumental con que los sujetos abordan la cuestión de la ciudadanía administrativa, ni siquiera el empadronamiento en el municipio de residencia aparece como una práctica común, excepto en caso de los sujetos que podemos vincular al discurso de la integración, esto es, aquellos en edad laboral que se establecen para trabajar, que generan un núcleo familiar y que tienen, sobre todo, hijos en edad escolar. El empadronamiento en estos casos responde esencialmente a los derechos que se 
conceden al estar empadronado, sobre todo en el ámbito educativo y socio-sanitario, lo que coincide con resultados de análisis realizados en otras comunidades autónomas con presencia relevante de extranjeros europeos (Rodríguez et al. 2010).

En términos de identidad nacional, parece que ambos discursos (el de la integración y el de la coexistencia) muestran un rechazo a la identificación con el nivel de Estadonación. El traslado o la itinerancia de su residencia les conduce a un desplazamiento de la identificación territorial, abandonando los marcos estatales y explicitando una vocación de identificación europeísta, autodefiniéndose como ciudadanos europeos, lo que fija sus anclajes territoriales en la formación del capital social sobre un espacio difuso, 0 cuando menos muy amplio. Aunque como podremos ver más adelante, si fijáramos una tipología de redes sociales en migraciones (véase De Federico 2004), nos hallaríamos ante perfiles mucho más cercanos al aislamiento que al cosmopolitismo.

Yo no me considero alemán sino europeo. (Hombre alemán, 51 años).

Ahora me siento más ciudadano europeo. (Hombre italiano, 42 años)

Una vez establecidos en la Comunidad Valenciana, los sujetos comienzan el proceso de inserción en la nueva sociedad receptora, y aquí también encontramos otro elemento común a ambos discursos, que además posee una importancia central para nuestro análisis. Sea cual sea el motivo y las condiciones de llegada, y al margen de la preparación previa, que en la mayoría de los casos es inexistente, los extranjeros europeos presentan una estrategia de inserción en la nueva sociedad con un carácter marcadamente individual. No cabe hablar de redes migratorias en el sentido clásico (Grasmuck y Pessar 1991), esto es, conjuntos de actores y vínculos establecidos entre la población extranjera, que organizan y dirigen la circulación de trabajo, capital, bienes, servicios, información e ideologías entre las comunidades que envían migrantes y las que los reciben. Ni siquiera podríamos conceptualizar las redes intensas que incluyan otros factores de sociabilidad, como relaciones de parentesco, amistad, o lazos difusos de sentido comunitario (Pedone 2006). En este sentido la inserción se despliega generalmente en ausencia o al margen de redes sociales, tanto formales como informales, bien de su comunidad de origen 0 bien ya existentes en el nuevo territorio que pudieran ayudar, orientar o catalizar este proceso. Los sujetos afrontan por tanto todos los cambios sociales y vivenciales que suponen el cambio de residencia desde la convicción de que la población de su misma nacionalidad que ya reside en el nuevo territorio no posee un rol especial en su proceso de inserción, y sin lamentar en ningún caso la escasa o nula ayuda que encuentran en las redes sociales de su comunidad de origen. La idea de la creación de capital social a partir de la generación de redes migratorias no responde en este caso a la dinámica de los extranjeros europeos, y se aleja de cualquier idea de construcción de realidades cercanas a lo que podríamos denominar capital social transnacional (Rippl et al. 2010). 
Ello incide de manera determinante en la concepción que desarrollan sobre el sentido de los lazos de confianza y convivencia en la comunidad de acogida.

El primer día ya encontré un piso, cogí el Fácil y llamé. (Mujer francesa, 26 años).

Yo vine a la aventura. No conocía a nadie. (Mujer francesa, 46 años).

Tuvimos que buscar tanto trabajo como casa por cuenta propia. (Mujer alemana 37 años).

Tampoco hemos pedido muchas ayudas a nadie. (Mujer francesa, 35 años).

Creemos conveniente resaltar esta situación porque supone una diferencia fundamental en relación con las estrategias que suelen acompañar los procesos migratorios de extranjeros extracomunitarios (Colectivo IOE y Fernández 2010). Ciertamente, el hecho de ser ciudadanos comunitarios facilita en parte el proceso, pero no parece un rasgo común el factor de atracción que puede suponer la inmigración "en cadena", derivada de la presencia ya en el territorio de acogida de familiares 0 amistades; un elemento que, por otra parte, se muestra relevante en los procesos migratorios no comunitarios (Aparicio y Tornos 2005). Tampoco hallamos referencias al contacto con redes sociales de la población de acogida como recurso para catalizar el proceso de inserción. La decisión del traslado de la residencia es de carácter individual y el itinerario que las personas siguen en el nuevo territorio también responde a estas coordenadas. En el caso de la población que acude en busca de ocupación laboral, esta estrategia individualizante está marcada en la mayoría de los casos por la existencia de nichos de trabajo específicos para los perfiles de población extranjera europea, pero las estrategias de búsqueda de empleo se desarrollan en ausencia de contactos en las redes sociales.

A partir de lo expresado hasta ahora hemos podido identificar dos formas de concebir la vida cotidiana por parte de los extranjeros europeos residentes en la Comunidad Valenciana, presididas ambas por una estrategia de inserción individual en la sociedad de acogida. Son estos discursos los que nos van a ayudar a comprender mejor el modo en que estos sujetos llevan a cabo la reconstrucción de su realidad social en el nuevo territorio, y cómo se configuran los principales elementos que reposicionan a los sujetos para la construcción de una nueva cotidianeidad. Y en este aspecto nos parece relevante tratar en detalle cuatro indicadores clave para la articulación del capital social.

La primera esfera es la que hace referencia al mantenimiento o cambio de "costumbres y tradiciones culturales" por parte de los residentes europeos en la nueva situación de residencia. Las costumbres y tradiciones constituyen un aspecto muy importante en el establecimiento de identificaciones, en la definición del sentimiento de pertenencia a una comunidad, marcando así referentes para comprender el grado de conservación de patrones culturales de la sociedad de origen y también el grado de adopción de nuevos patrones propios de la sociedad de acogida. En relación a ello podemos concluir, a la luz de la información derivada de los sujetos entrevistados, que el mantenimiento 
de las tradiciones de origen es común a ambos discursos, pero se revela con una fuerza relativamente débil. La conservación de las tradiciones parece que se reduce esencialmente a la reproducción de estereotipos o festividades puntuales del calendario. Los nexos con la sociedad de origen cristalizan en elementos religiosos o determinadas fechas de especial significación en el país de origen. Únicamente el mantenimiento de la lengua aparece con un fuerte vínculo cultural con su país, pero desprovisto generalmente de atribuciones de identidad, no encontrándose argumentaciones que liguen la lengua con sentimientos de pertenencia.

Respecto a la adopción por parte de la población extranjera de tradiciones españolas, podemos afirmar que se sitúan en el mismo plano de importancia que las tradiciones de la comunidad de origen. Las costumbres españolas son percibidas positivamente en tanto que se les atribuyen componentes lúdicos 0 de mejora de la calidad de vida. En este sentido, sobre todo en el caso del discurso de la integración, encontramos que se produce una adaptación favorable a los horarios españoles, la gastronomía 0 la presencia de vida social en el espacio público, pero en cualquier caso constituyen elementos que no suponen un activo relevante en el proceso de integración social o de sentimiento de pertenencia a la nueva comunidad de acogida.

La siguiente esfera que incide en la reorganización de la realidad de los residentes europeos es la que nos habla de las "redes sociales y afectivas" que mantienen con su comunidad de origen. En relación a ello, emerge como cuestión común el debilitamiento y abandono progresivo de los lazos afectivos que los sujetos mantenían con los círculos de pertenencia tanto familiares como de amistad en su país de origen.

Mi familia vive toda en Inglaterra. Tengo dos hermanos, dos hermanas, pero no los veo mucho. (Hombre inglés, 55 años).

Ahora que vivo aquí mi realidad es esta, y es difícil que piense en los amigos de Italia (Hombre italiano, 42 años).

El cambio de residencia aparece como un elemento de ruptura que configura una nueva forma de vida de los sujetos residentes, no solo en tanto que cambio de costumbres y entorno, sino también en aquellos aspectos propios de la esfera afectiva. Se trata, como es de suponer, de redes que contribuyen de un modo determinante a dar forma al entramado de identificaciones de las personas, y su debilitamiento o desarticulación nos ayudan a comprender la laxitud de los discursos al enunciar sus claves identitarias como sujetos. En este sentido, además, el abandono de las redes sociales preexistentes al cambio de residencia no parece ser sustituido por nuevas relaciones relevantes en el nuevo entorno social, a lo cual contribuye sin duda la asunción de una estrategia de inserción individual situada al margen de la comunidad de origen. En el mejor de los casos las nuevas redes están integradas, en el caso del discurso de la integración, por 
relaciones afectivas de pareja y por relaciones débiles en el ámbito laboral, y en el caso del discurso de la coexistencia por redes débiles con la población residente de la misma nacionalidad ligadas al territorio.

Otra de las esferas alusivas a la reconstrucción de la realidad social es la vinculada a la "participación social y ciudadana" en el nuevo espacio de residencia. A través del discurso de los sujetos podemos establecer diferencias entre las formas de participación social formal (asociacionismo y participación política) y las formas de participación social informal (vinculada a eventos sociales pero realizada de forma no organizada).

En lo referente a la participación política, podríamos calificar la actividad de los residentes europeos como de baja implicación y relativo desconocimiento de la realidad política española, lo que les conduce con frecuencia a desarrollar conductas de inhibición electoral. Manifiestan poco interés por la política y desconocen en muchos casos sus derechos políticos. Esta desafección se encuentra, si no originada, al menos sí acompañada por los problemas burocráticos que acarrea el deseo de ejercer sus derechos como votantes. El hecho de tener que llevar a cabo trámites burocráticos y el desconocimiento de estos para poder votar supone un esfuerzo que en muchos casos termina por acentuar la desafección por los mecanismos electorales, lo que liga de nuevo el discurso de los residentes extranjeros con la crítica hacia la Administración española, aunque por lo general los sujetos manifiestan poco interés personal por incorporarse como votantes efectivos.

No me apunté al censo electoral por el papeleo. No lo sé, yo para esas cosas estoy perdida (Mujer alemana, 28 años).

No he arreglado los papeles para votar. (Mujer inglesa, 42 años).

Al margen de la participación política, podemos evaluar los posicionamientos de los sujetos ante el mundo asociativo o de participación organizada. Dentro de este espacio cívico hallamos una integración relativamente baja. La asociatividad, cuestión central para valorar la cuestión del capital social en los residentes europeos, se muestra débil, tanto en lo referente a la participación de extranjeros en la vida asociativa de la comunidad de acogida como en la creación y desarrollo de asociaciones con membresía extranjera, lo cual viene a coincidir con otros estudios que han tratado este tema para la población extranjera en general (Colectivo loé y M. Fernández 2010:207). Las opiniones hacia el mundo de la participación organizada basculan entre lo que podríamos llamar asociacionismo pragmático (de carácter instrumental), la implicación a partir de preferencias individuales (como satisfactor de necesidades individuales de los sujetos en el amplio abanico de sectores asociativos), o bien la crítica generalizada hacia las organizaciones sociales, que son escasamente valoradas por parte de los entrevistados. En cualquier caso el cambio de residencia no parece suponer ni un inhibidor ni un 
catalizador de los patrones de filiación asociativa. De hecho, se registra más participación en entidades ciudadanas orientadas a la población en general (asociaciones de vecinos, urbanísticas, culturales, medioambientales, las AMPA, etc), participación que nos habla de una sociabilidad exogrupal (Torres 2005) o conexión extracomunitaria (Massanet y Santacreu 2010), que a las específicas para personas de origen extranjero, lo que vendría a dar cuenta de formas de sociabilidad endogrupal o integración intracomunitaria. Dicho de otra manera, si los individuos eran activos en asociaciones en el territorio de origen buscarán vías de continuidad en la nueva residencia, pero, si no lo eran, la nueva situación vital tras el traslado no supone en ningún caso un reclamo para la implicación en colectivos ciudadanos. Además, los rasgos de participación registrados a través de los discursos de los entrevistados dejan entrever una participación de carácter sectorial, finalista, ligada a afinidades personales 0 a la necesidad percibida de cubrir satisfactoriamente determinadas necesidades, lo que coincide con otras investigaciones sobre asociacionismo de extranjeros comunitarios (Gómez 2006; Simó et al. 2005). Se abre así una visión individual e instrumental de la participación, concordante con la visión particularista del proceso de inserción social, pero no una participación ligada al territorio que se habita, vinculada a las características o problemáticas del nuevo espacio en que se vive.

Hombre, como residente yo estoy bien. Sé que hay extranjeros en Torrevieja o por esa zona que sí que participan. (Mujer alemana, 28 años).

he participado también el Alemania en acciones de los verdes. Eso en Alemania. Aquí he participado [...] aquí políticamente no he hecho nunca nada. Quiero, quiero ir a esto, a Ecologistas en Acción, pero fui una vez que tenían cerrado su local, esa sería mi participación, esa es mi idea. (Hombre alemán, 55 años).

Esta forma de participar, básicamente a partir de nexos con aficiones personales, dibuja un mapa participativo en el que lo lúdico y lo festivo presiden el contenido de la actividad asociativa.

Pertenezco a una asociación de fútbol, donde se pueden hacer muchos amigos, yo fui uno de los creadores de esta asociación. (Hombre inglés, 45 años)

Sí, sí. Yo soy costalero. Estoy en una cofradía internacional en Torrevieja. (Hombre inglés, 57 años).

De esta forma, el perfil de participación, en tanto que se liga a las prácticas de ocio, a elementos festivos, se traslada en mayor medida (sobre todo en el discurso de la coexistencia) hacia canales de participación informal, vinculada a la fechas de festividades del nuevo territorio, suponiendo éste el principal espacio de contacto con la población 
española. Hallamos aquí nexos con la evaluación positiva en términos de vitalidad que atribuyen a la vida en España, que maximiza según los sujetos las posibilidades de ocio colectivo. Al margen de ello, si tenemos que aludir a alguna forma adicional de participación, cabe señalar la implicación esporádica de los sujetos en acciones reivindicativas sumándose a la comunidad española, pero siempre con carácter puntual y con una participación escasamente estructurada.

Las fiestas son realmente fiestas, más animadas. Porque en Francia, todo ha muerto, porque quieren siempre demasiada seguridad, demasiada autorización, y entonces ya no se puede hacer nada sin autorización.... (Mujer francesa, 26 años).

Lo que me gusta más es que aquí hay más vida, la gente es más...aquí hay más fiesta, hay más espectáculos. (Mujer francesa, 34 años).

Nunca he participado en una asociación o un partido. En asociaciones culturales sí. Luego manifestaciones con pancartas y cosas así cuando era la hora... eso fundamentalmente. (Hombre italiano, 59 años).

Finalmente, podemos entrar al detalle en una cuarta esfera que contribuye en gran medida a los procesos de reconstrucción de la realidad social que realizan los residentes extranjeros en su nueva residencia: es la esfera del papel de los "medios de comunicación" en este proceso. En relación a ello, cabe resaltar que los medios de comunicación no ejercen una función apreciable en la construcción de realidades propias de los sujetos entrevistados ni en su vinculación a la sociedad civil dentro del nuevo territorio. En referencia al seguimiento de medios de comunicación extranjeros, lo más resaltable es que su consumo parece deberse más a los formatos y contenidos que presentan los medios que a su posible función como elemento de identificación con la comunidad de origen. Respecto al formato y contenido de los medios, explicitan en todo momento diferencias sensibles entre los medios de comunicación del país de origen y los españoles, valorados estos últimos negativamente por su poca seriedad, lo que les lleva generalmente a atribuirles una credibilidad muy escasa en la formación de opiniones ante la realidad social.

Lo que pasa con los medios de comunicación españoles es una pasada. Están supersalidos. Eso es imposible ahora en Francia. (Mujer francesa, 35 años).

A pesar de estas diferencias el seguimiento de los medios, sean extranjeros o españoles, es bastante bajo y su uso esporádico, por lo que cabe cuestionar la incidencia de estos en la realidad social que pretendemos abordar en este texto. 


\section{Conclusiones}

Recordemos brevemente al inicio del apartado de conclusiones las hipótesis investigadoras que sometíamos a examen en este texto. La primera intentaba dar cuenta de la existencia de lo que podríamos calificar como laxitud de las señas identitarias en los itinerarios de inserción de la población extranjera comunitaria, alejando la comprensión del fenómeno de las claves explicativas vinculadas a variables tradicionales propias de la formación de capital social. Una segunda hipótesis hacía alusión a que la explicación de esta difuminación identitaria descansaría en una estrategia de socialización con un marcado componente individual, al margen de las redes sociales de la población de su misma procedencia o ya existentes en el territorio de acogida, las cuales también suponen recursos muy presentes en los estudios sobre capital social. Y finalmente una tercera hipótesis hacía referencia a la existencia de diferencias internas significativas en el proceso de integración, a pesar de los elementos de socialización compartidos en la población extranjera europea, diferencias que se explicarían primordialmente por la edad de llegada de la persona al nuevo territorio.

Los elementos comunes en los discursos de la población extranjera comunitaria confirman positivamente la primera hipótesis planteada en este trabajo, en la que se hacía alusión a la "laxitud de señas identitarias" habituales en el estudio de los procesos migratorios, como son la nacionalidad, la lengua o las tradiciones, elementos inherentes al desarrollo tradicional de capital social en la población inmigrante. Como hemos podido apreciar, estas variables resultan escasamente significativas en la articulación de los discursos de los sujetos entrevistados para comprender sus estrategias de socialización. Los resultados muestran además el hecho de que la población extranjera comunitaria inmigrante comparte una concepción individualizante del proceso de inserción en la sociedad civil del nuevo territorio de residencia, lo que confirma la segunda hipótesis de trabajo, así como una consideración instrumental de la nacionalidad por parte de los sujetos. Estos parámetros se alejan sustancialmente de las claves explicativas en los itinerarios de inserción en la sociedad civil que registran la mayor parte de las investigaciones de este tipo planteadas con población extranjera extracomunitaria, y que dan cuenta de la formación de capital social en la población inmigrante.

Los tres elementos citados (la debilidad de las señas identitarias, el proceso autónomo de inserción y la visión instrumental de la nacionalidad) revelan la base de las diferentes dimensiones de la sociabilidad intragrupal y exogrupal dentro del contingente de inmigrantes extranjeros europeos, y por consiguiente, los mecanismos particulares de construcción de capital social en esta población. Condicionan el mantenimiento 0 modificación de las costumbres y tradiciones previas, dan cuenta de la debilidad o el desmantelamiento en algunos casos de las redes sociales de las que forman parte, explican la funcionalidad instrumental del sentido otorgado a la participación asociativa 
y dilucidan el escaso papel que juegan los medios de comunicación en los procesos de reconstrucción de la realidad social dentro del nuevo territorio.

Poniendo en relación los resultados de las hipótesis y las dimensiones del capital social del modelo de Woolcock, se pone de manifiesto un bajo grado de integración intracomunitaria y conexión extracomunitaria, así como una escasa sinergia institucional y reducida eficiencia organizativa. En lo referente a la "integración intracomunitaria", las estrategias de integración de los extranjeros europeos evidencian una escasa participación asociativa en las propias organizaciones de extranjeros, y un abandono progresivo de las tradiciones, costumbres y redes sociales en los países de origen, así como una debilidad en la generación de redes sociales significativas con población de su procedencia dentro del nuevo territorio, al margen de elementos catalizadores de cohesión intragrupal como son la defensa corporativa de interés o la reivindicación institucional. En relación a la "conexión extracomunitaria" nos encontramos también ante una situación de inhibición en la participación dentro de la comunidad de acogida, si exceptuamos las redes afectivas generadas a partir de la formación de parejas o familias transnacionales; la integración asociativa o la participación sociopolítica registra hoy por hoy un escaso interés por parte de los europeos extranjeros. Relacionado con ello, cabe hablar también de un bajo nivel de "sinergia institucional". Hay una débil articulación asociativa entre las organizaciones de inmigrantes, que se caracterizan más por su aislamiento que por la coordinación de sus intereses. Esto, unido a la inserción marginal de la población extranjera europea en los sectores asociativos ya existentes de la sociedad de acogida (organizaciones vecinales, políticas, sindicales, cívicas, culturales, etc.), condicionaría la formación y articulación de capital social en la Comunidad Valenciana. En lo referente a la dimensión de la "eficiencia organizativa", podemos destacar a través de los resultados obtenidos la percepción negativa del entramado institucional y administrativo español. La inadecuada implicación de la administración pública en el proceso de asentamiento y consolidación del proyecto migratorio individual se manifiesta en una serie de deficiencias percibidas en el tratamiento institucional, lo que ayuda bien poco al establecimiento de puentes colaborativos entre esta población y las instituciones públicas. El éxito o fracaso de las estrategias de integración descansa primordialmente en las competencias y habilidades individuales, más que en la expectativa de asistencia de redes informales 0 institucionales de apoyo.

Finalmente, en relación a nuestra tercera hipótesis, la que hacía referencia a la existencia de fracturas o diferencias internas en los discursos ligados a las estrategias de socialización, el análisis realizado nos ha mostrado dos formas o vías a través de las cuales es posible comprender mejor las estrategias que la población europea residente adopta en su proceso de inserción en la sociedad civil de la comunidad de acogida: el discurso de la integración y el discurso de la coexistencia. Constituyen dos caminos distintos que vienen a representar predisposiciones, necesidades y conductas diferentes. El primero se halla representado en un posicionamiento caracterizado por el 
reconocimiento del deber de integración en la sociedad de acogida, adoptando nuevos patrones de sociabilidad y pautas relacionales. El segundo, sin embargo, explicita el derecho a coexistir con la sociedad de acogida, sin tener que adoptar necesariamente nuevos patrones culturales que les conduzcan a la integración social. El desarrollo de uno u otro discurso, como hemos podido apreciar, depende más de variables demográficas y socioeconómicas, como son la edad y la posición laboral, que del despliegue diferencial por parte de los sujetos de los elementos propios del capital social.

Por lo visto a lo largo del análisis de la información, la formación de capital social y las estrategias de inserción en la sociedad civil de los extranjeros europeos componen un paradigma explicativo de muy distinta naturaleza a la de subyace en los procesos de inmigración extracomunitaria. Pero en nuestra opinión merece tenerse en cuenta si desde cualquier ámbito de estudio se persigue contemplar este creciente contingente de población a efectos de planteamientos analíticos. Una población que, por otro lado, está llamada a ejercer una influencia determinante en la dinámica social de los próximos años no solo en la Comunidad Valenciana, sino también en otros territorios del Estado español.

Más allá de los resultados y conclusiones investigadoras, el tema tratado en este texto plantea sugerencias de continuidad o profundización para futuros estudios. Quizás la principal línea investigadora a abordar sea el análisis longitudinal y la evolución del proceso a lo largo del tiempo, máxime en un contexto como el actual en trance de reordenación multidireccional de los flujos migratorios. Se muestra conveniente, además, señalar la necesidad de acrecentar el número de investigaciones dedicadas a analizar con mayor profundidad la inserción y participación de las personas inmigrantes en el tejido asociativo autóctono, al margen de las organizaciones sociales endógenas propias de la comunidad extranjera, lo cual contribuirá sin duda a dar una mejor cuenta del alcance de los procesos de integración.

Por terminar con una cierta voluntad de síntesis, situamos al final de este artículo la información tratada a modo de modelo de análisis relacional (Figura 4). Lejos de pretender constituir un sistema cerrado, el modelo intenta mostrar, de manera gráfica, los principales discursos encontrados en el análisis, así como las relaciones que se establecen entre dichos discursos y los elementos ligados a ellos considerados como relevantes en la formación de capital social de la población extranjera europea residente en la Comunidad Valenciana. 


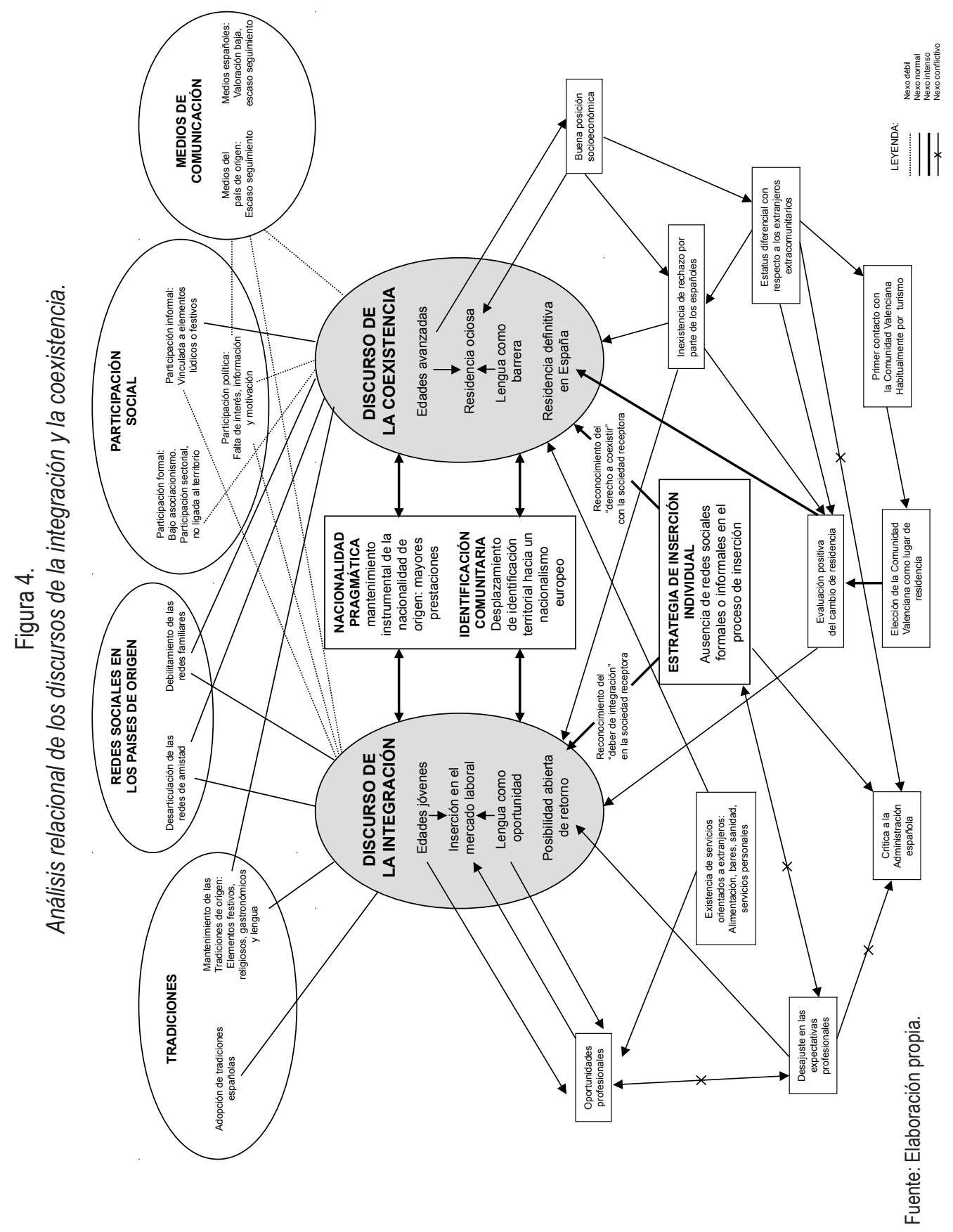




\section{RefERENCIAS BibliográficAS}

Aparicio, R. y A. Tornos. 2010. Las asociaciones de inmigrantes en España: una visión de conjunto. Madrid: Ministerio de Trabajo e Inmigración.

Aparicio, R. y A. Tornos. 2005. Las redes sociales de los inmigrantes extranjeros en España. Madrid: Ministerio de Trabajo y Asuntos Sociales.

Banco Mundial. 1999. How is social capital measured? The World Bank: Washington. Fecha de consulta: 10/09/2010 (http://www.worldbank.org/poverty/scapital).

Bourdieu, P. 1998. Practical reason. On the Theory of Action. Cambridge: Polity.

Bourdieu, P. 1985. "The forms of Capital". Pp. 241-258 in Handbook of theory and research for the Sociology of Education, edited by J. G. Richardson. Connecticut: Greenwood Press.

Colectivo IOE y M. Fernández. 2010. Encuesta nacional de inmigrantes 2007: el mercado de trabajo y las redes sociales de inmigrantes. Madrid: Ministerio de Trabajo e Inmigración.

Coleman, J. 1988. "Social capital in the creation of human capital." American Journal of Sociology 94:95-120.

De Federico, A. 2004. "Los espacios sociales de la transnacionalidad. Una tipología de integración relacional de los inmigrantes". Redes. Revista hispana para el análisis de redes sociales 4.

Díez Nicolás, J. 2005. Las dos caras de la inmigración. Madrid: Ministerio de Trabajo y Asuntos Sociales.

Favell, A. 2003. "Games without frontiers? Questioning the transnational social power of migrants in Europe." Archives Européennes de Sociologie 3:106-136.

Fine, B. 2001. Social capital versus social theory. Political economy and social science at the turn of the millenium. London and New York: Rotledge.

Gómez, C. 2006. "Inmigración y asociacionismo: el caso de Alicante". Pp. 1-19 en Otras miradas sobre la inmigración, coordinado por C. Gómez. Alicante: Universidad de Alicante.

Grasmuck, S. y P. Pessar. 1991. Between Two Islands. Dominican International Migration. California: University of California Press.

Jabbaz, M. et al. 2004. "Los inmigrantes y el mundo asociativo en la Comunidad Valenciana: una reflexión en torno a las formas de participación y las funciones sociales", IV Congreso de migraciones, Girona.

Martín, A. 2004. "Las asociaciones de inmigrantes en el debate sobre las nuevas formas de participación política y de ciudadanía: reflexiones sobre algunas experiencias en España". Revista Migraciones 15:113-143. 
Martín-Hernández, P., E. Ochoa, A. Izquierdo y M. Gil-Lacruz. 2007. "Capital social e inmigración: conceptualización operativa de la inserción sociolaboral de los inmigrantes". Revista de Humanidades 13:257-281.

Massanet y Santacreu 2010. "El movimiento asociativo inmigrante en la Comunidad Valenciana y sus repercusiones en la producción de capital social". Revista Migraciones 27:49-81.

Massey, D. S. y K. E. Espinosa. 1997. "What's Driving Mexico-U.S. Migration? A Theoretical, Empirical, and Policy Analysis." American Journal of Sociology 102 4:939-999.

Migallón, J. 2004. "Breves apuntes para un análisis del movimiento asociativo en migración en España". IV Congreso de migraciones. Girona.

Miravet, P. 2006. "Algunos problemas para la participación cívica y política de los inmigrantes". Cuadernos electrónicos de filosofía del derecho 14.

Morales L., Anduiza, E., Rodríguez, E. y J. San Martín. 2008. "Capital social, pautas identitarias y actitudes hacia 'los otros': la incorporación cívica de la población de origen inmigrante en Barcelona y Madrid". Panorama Social 8:119-142.

Morell, A. 2005. "El papel de las asociaciones de inmigrantes en la sociedad de acogida: cuestiones teóricas y evidencia empírica". Revista Migraciones 17:11-42.

Moyano, E. 2006. "El asociacionismo en el sector agroalimentario y su contribución a la generación del capital social". IESA Working Paper Series 20-06. CSIC. Córdoba.

Pedone, C. 2006. "Tú siempre jalas a los tuyos. Cadenas y redes migratorias de las familias ecuatorianas hacia España". Pp. 105-146 en La migración ecuatoriana. Transnacionalismo, redes e identidades Herrera, editado por Herrera, G. et al.; México: FLACSO.

Portes, A. 1995. "The Economic Sociology and Sociology of Immigration: a Conceptual Review". Pp. en The Economic Sociology of Immigration: Essays on Networks, Ethnicity and Entrepreneurship, edited by A. Portes. N.Y: Rusell Sage Foundation.

Portes, A. 1998. "Social capital. Its origins and applications in modern sociology". Annual Review of Sociology 24:1-24.

Putnam, R. 1995. "Bowling alone". Journal of Democracy 6, 1:65-78.

Putnam, R. 2000. Bowling Alone: The Collapse and Revival of American Community. New York: Simon \& Schuster.

Putnam, R., R. Leonardi y R. Nanetti. 1993. Making Democracy Work. Civic Traditions in Modern Italy. Princeton: Princeton Univ. Press.

Rippl et al. 2010. "Crossing the frontier: Transnational Social integration in the EU's Border Regions". International Journal of Comparative Sociology 1:5-31. 
Rodríguez, V. Lardiés, R. y P. Rodríguez. 2010. La migración y el registro de jubilados europeos en España. Madrid: Real Instituto Elcano.

Simó, C. Jabbaz, M. y J. Giner Monfort. 2005. "Asociacionismo y población extranjera en la Comunidad Valenciana”. Cuadernos electrónicos de filosofía del derecho 12:1-57.

Torres, F. 2005. Ámbit urbà, sociabilitat i inserció social dels immigrants. El cas de Russafa València., Valencia. Tesis doctoral, Universitat de València.

Veredas, S. 2003. "Las asociaciones de inmigrantes en España. Práctica clientelar y cooptación política." Revista internacional de sociología 36:207-235.

Vidal, P. et al. 2007. "Diagnóstico sobre las asociaciones de personas inmigradas en España", $V$ Congreso de Migraciones, Valencia.

Woolcock, M. 1998. "Social capital and economic development: toward a theoretical synthesis and policy framework." Theory and Society 2:151-208.

Francisco José Francés García es Doctor en Sociología y premio extraordinario de doctorado por la Universidad de Alicante. En la actualidad es profesor en el Departamento de Sociología II de la UA. Asimismo, es miembro investigador del Instituto Interuniversitario de Desarrollo Social y Paz. Sus principales líneas de investigación son la participación social, sociología política, migraciones y juventud, áreas en las que ha participado en más de una veintena de proyectos públicos.

Órcar Antonio Santacreu Fernández es Profesor en el Departamento de Sociología II de la Universidad de Alicante y miembro del Instituto Interuniversitario de Desarrollo Social y Paz (UAUJI). Investigador principal en proyectos competitivos de I+D+I europeos, nacionales y autonómicos. Sus principales líneas de investigación son los métodos y técnicas de investigación social, procesos migratorios intraeuropeos, la música como elemento performativo en los procesos de comunicación social y el desarrollo de material docente y software para la docencia y la investigación.

RECIBIDO: 24/05/2011

ACEPTADO: 18/05/2011

Publicado on-line: 12/11/2012 